\title{
Evidence-Based Clustering of Reads and Taxonomic Analysis of Metagenomic Data
}

\author{
Gianluigi Folino $^{1}$, Fabio Gori ${ }^{2}$, Mike S.M. Jetten ${ }^{2}$, and Elena Marchiori ${ }^{2, \star}$ \\ 1 ICAR-CNR, Rende, Italy \\ 2 Radboud University, Nijmegen, The Netherlands \\ elenam@cs.ru.nl
}

\begin{abstract}
The rapidly emerging field of metagenomics seeks to examine the genomic content of communities of organisms to understand their roles and interactions in an ecosystem. In this paper we focus on clustering methods and their application to taxonomic analysis of metagenomic data. Clustering analysis for metagenomics amounts to group similar partial sequences, such as raw sequence reads, into clusters in order to discover information about the internal structure of the considered dataset, or the relative abundance of protein families. Different methods for clustering analysis of metagenomic datasets have been proposed. Here we focus on evidence-based methods for clustering that employ knowledge extracted from proteins identified by a BLASTx search (proxygenes). We consider two clustering algorithms introduced in previous works and a new one. We discuss advantages and drawbacks of the algorithms, and use them to perform taxonomic analysis of metagenomic data. To this aim, three real-life benchmark datasets used in previous work on metagenomic data analysis are used. Comparison of the results indicates satisfactory coherence of the taxonomies output by the three algorithms, with respect to phylogenetic content at the class level and taxonomic distribution at phylum level. In general, the experimental comparative analysis substantiates the effectiveness of evidence-based clustering methods for taxonomic analysis of metagenomic data.
\end{abstract}

\section{Introduction}

The rapidly emerging field of metagenomics seeks to examine the genomic content of communities of organisms to understand their roles and interactions in an ecosystem. Given the wide-ranging roles microbes play in many ecosystems, metagenomics studies of microbial communities will reveal insights into protein families and their evolution. Because most microbes cannot grow in the laboratory using current cultivation techniques, scientists have turned to cultivationindependent techniques to study microbial diversity. At first shotgun Sanger sequencing was used to survey the metagenomic content, but nowadays massive parallel sequencing technology like 454 or Illumina, allow random sampling of DNA sequences to examine the genomic material present in a microbial community [1].

\footnotetext{
^ Corresponding author.
} 
For a given sample, one would like to determine the phylogenetic provenance of the obtained fragments, the relative abundance of its different members, their metabolic capabilities, and the functional properties of the community as a whole. To this end, computational analysis is becoming increasingly indispensable 23]. In particular, clustering methods are used for rapid analysis of sequence diversity and internal structure of the sample [4, for discovering protein families present in the sample [5], and as a pre-processing set for performing comparative genome assembly [6], where a reference closely related organism is employed to guide the assembly process.

In this paper we focus on clustering methods and their application to taxonomic analysis of metagenomic data. Clustering analysis for metagenomics amounts to group similar partial sequences, such as raw sequence reads, or candidate ORF (Open Reading Frame) sequences generated by an assembly program into clusters in order to discover information about the internal structure of the considered dataset, or the relative abundance of protein families. Different methods for clustering analysis of metagenomic datasets have been proposed, which can be divided into two main approaches. Sequence- and evidence-based methods.

Sequence-based methods compare directly sequences using a similarity measure either based on sequence overlapping [4] or on extracted features such as oligonucleotide frequency [7. Evidence-based methods employ knowledge extracted from external sources in the clustering process, like proteins identified by a BLASTx search (proxygenes) [5]. In this paper we focus on the latter approach for clustering short reads.

We consider two clustering algorithms introduced in previous works [5] and a refinement of the latter one based on ensemble techniques. These algorithms cluster reads using weighted proteins as evidence. Such proteins are obtained by a specialized version of BLAST (Basic Local Alignment Search Tool), called BLASTx, which associates a list of hits to one read. Each hit consists of one protein, two score values, called bit and identities, which measure the quality of the read-protein matching, and one confidence value, called E-value, which amounts to a confidence measure of the matching between the read and the protein.

Specifically, in [5] an algorithm, here called LWproxy (Local Weight proxy), is introduced, that clusters reads and those proteins in their sets of hits simultaneously, in such a way that one cluster of proteins is associated to one cluster of reads. Then it assigns one local weight to each protein of a cluster, using the cumulative BLASTx bit score of those reads in corresponding cluster having that protein as one of their hits. The protein with best weight (highest cumulative bit score) is selected as proxygene of the cluster of reads.

In [8, an alternative method for clustering metagenome short reads based on weighted proteins is proposed, here called GWproxy (Global Weight proxy). The method first assigns global weights to each protein using the BLASTx identity and bit score of those reads having that protein as one of their hits. Next, the method groups reads into clusters using an instance of the weighted set covering 
problem, with reads as rows and proteins as columns. It seeks the smallest set of columns covering all rows and having best total weight. A solution corresponds to a clustering of reads and one protein (proxygene) associated to each cluster.

While in [5] the proxygene of a cluster is selected within a set of proteins associated to that cluster, in GWproxy clustering and proxygene selection are performed at the same time.

In this paper we introduce a refinement of GWproxy based on the following ensemble technique, called EGWproxy (Ensemble Global Weight proxy). The algorithm associates a list of proteins to each cluster resulting from application of GWproxy, such that each protein occurs as hit of each of the reads of that cluster. Such a list is used for refining the biological analysis of the cluster, for instance by assigning a taxonomic identifier (taxID) by means of weighted majority vote among the taxID's of the proteins in the associated list.

We discuss advantages and drawbacks of the above clustering algorithms, and use them to perform taxonomic analysis of metagenomic data. To this aim, three real-life benchmark datasets used in previous work on metagenomic data analysis are used. These datasets were introduced in [5] and used to perform a thorough analysis of evidence-based direct and indirect (that is, using proxygenes) annotation methods for short metagenomic reads. The results of such analysis substantiated advantages and effectiveness of indirect methods over direct ones.

The results of the three considered evidence-based clustering algorithms indicate satisfactory coherence of the taxonomies output by the algorithms. In general, the experimental comparative analysis substantiates the effectiveness of evidence-based methods for taxonomic analysis of metagenomic data.

\section{Clustering Metagenome Short Reads Using Proxygenes}

Different methods for clustering analysis of metagenomic datasets have been proposed, which can be divided into two main approaches. Sequence- and evidencebased methods. Sequence-based methods compare directly sequences using a similarity measure either based on sequence overlapping [4 or on extracted features such as oligonucleotide frequency [7. Evidence-based methods employ knowledge extracted from external sources in the clustering process, like proteins identified by a BLASTx search (proxygenes) [5].

Here we consider the latter approach for clustering short reads. The knowledge used by the clustering algorithms here considered is extracted by a reference proteome database by matching reads to that database by means of BLASTx, a powerful search program. BLASTx belongs to the BLAST (Basic Local Alignment Search Tool) family, a set of similarity search programs designed to explore all of the available sequence databases regardless of whether the query is protein or DNA 910. BLASTx is the BLAST program designed to evaluate the similarities between DNA sequences and proteins; it compares nucleotide sequence queries dynamically translated in all six reading frames to peptide sequence databases. The scores assigned in a BLAST search have a statistical interpretation, making real matches easier to distinguish from random background hits. In the following we summarize the main features of BLAST. 


\subsection{The BLAST Alignment Method}

BLAST uses a heuristic algorithm that seeks local as opposed to global alignments and is therefore able to detect relationships among sequences that share only isolated regions of similarity [11. When a query is submitted, BLAST works by first making a look-up table of all the words (short subsequences, three letters in our case) and neighboring words, i.e., similar words in the query sequence. The sequence database is then scanned for these strings; the locations in the databases of all these words are called word hits. Only those regions with word hits will be used as alignment seeds. When one of these matches is identified, it is used to initiate gap-free and gapped extensions of the word. After the algorithm has looked up all possible words from the query sequence and extended them maximally, it assembles the statistically significant alignment for each query-sequence pair, called High-scoring Segment Pair (HSP).

The matching reliability of read $r$ and protein $p$ is evaluated trough Bit Score, denoted by $S_{B}(r, p)$, and $E$-value, denoted by $E(r, p)$. The bit score of one HSP is computed as the sum of the scoring matrix values for that segment pair. The E-value is the number of times one might expect to see such a query-sequence match (or a better one) merely by chance. Another important BLASTx score of matching between $r$ and $p$ is Identities score, denoted by $\operatorname{Id}(r, p)$, defined as the proportion of the amino-acids in the database sequence $p$ that are matched by the amino-acids translation of the current query frame $r$. We refer to 9 for a formal description of these measures.

We turn now to describe the three methods here used for taxonomic analysis of metagenomic data. Here and in the sequel we assume the BLASTx has been applied to a metagenomic data set with a given Evalue cutoff value. We denote by $R=\left\{r_{1}, \ldots, r_{m}\right\}$ the resulting set of reads having at least one BLASTx hit for the given cutoff, and by $P=\left\{p_{1}, \ldots, p_{n}\right\}$ the set of proteins occurring in the hit of at least one read of $R$.

\subsection{LWproxy}

LWproxy generates a collection of pairs $\left(C_{i}, P_{i}\right)$, where $C_{i}$ is a set of reads and $P_{i}$ a set of proteins. The algorithm can be summarized as follows.

1. Set $i=0$.

2. Set $X=R$.

3. If $X$ is empty then terminate, otherwise set $i=i+1$.

4. Select randomly ${ }^{1}$ one read $r_{i}$ from $X$ as seed of cluster $C_{i}=\left\{r_{i}\right\}$.

5. Set $P_{i}$ to the set of hits of $r_{i}$.

6. Remove $r_{i}$ from $X$.

7. Add to $C_{i}$ all the reads having one element of $P_{i}$ as a best hit, and remove them from $X$.

8. Add to $P_{i}$ all hits of those reads added to $C_{i}$ in the previous step.

9. If no reads are added then go to step 3 , otherwise go to step 7 .

${ }^{1}$ We consider here random seed selection. However, in [5] the criterion for selecting a seed is not specified. 
When the clustering process is terminated, the method assigns one proxygene to each $C_{i}$ by selecting from $P_{i}$ the protein having highest cumulative bit-score.

Example 1. Suppose given a set of five reads $\left\{r_{1}, \ldots, r_{5}\right\}$ and suppose that the proteins occurring in their hits:

$-\left\{p_{1}, p_{3}, p_{5}\right\}$ for read $r_{1}$, with best hit $p_{3}$.

$-\left\{p_{1}, p_{3}, p_{5}\right\}$ for read $r_{2}$, with best hit $p_{3}$.

$-\left\{p_{2}, p_{4}\right\}$ for read $r_{3}$, with best hit $p_{2}$.

$-\left\{p_{2}\right\}$ for read $r_{4}$, with best hit $p_{2}$.

$-\left\{p_{2}, p_{3}, p_{5}\right\}$ for read $r_{5}$, with best hit $p_{2}$.

If LWproxy starts from $r_{1}$ as seed for $C_{1}$ then only $r_{2}$ is added to $C_{1}$, since $p_{2}$ (best hit of each of the other reads) does not occur in the list of protein associated to $C_{1}$. Then construction of a second cluster, say $C_{2}$ begins. $C_{2}$ is filled with the rest of the reads $\left\{r_{3}, r_{4}, r_{5}\right\}$.

\subsection{GWproxy}

While LWproxy constructs clusters incrementally, GWproxy searches for clusters in a given search space, consisting of clusters characterized by the proteins as follows. We say that a protein covers a read if the protein occurs as one of the hits of that read. Then each protein characterizes one cluster, consisting of the reads it covers. Moreover, we can assign to each protein a global weight, representing the cost of selecting that protein as cluster representative. The weight of protein $p$ is defined as

$$
w(p)=1+\left\lceil\frac{1}{N_{p}} \sum_{r \mid p \text { hit of } r}\left(100 \frac{S_{B}^{\max }-S_{B}(r, p)}{S_{B}^{\max }-S_{B}^{\min }}+100-I d(r, p)\right)\right\rceil,
$$

where $\ulcorner v\urcorner$ denotes the smallest integer bigger or equal than $v$, and $N_{p}$ is the number of reads having $p$ as one of their hits. The maximum and minimum value of $S_{B}$ over the considered pairs of reads and proteins, $S_{B}{ }^{\max }$ and $S_{B}{ }^{\min }$, respectively, are used to scale $S_{B}(r, p)$. The weight $w(p)$ is in inverse proportion to the average quality of the matchings between $p$ and the reads having $p$ as a hit, so that better proteins have smaller $w(p)$ value (smaller cost).

Clustering then amount at finding a minimum set of proteins in $R$ that, together, cover all the reads in $R$ and have minimum total cost. Formally, consider the vector of protein weights $w \in \mathbb{N}^{n}$ and the matrix $A \in\{0,1\}^{m \times n}$ whose elements $a_{i j}$ are such that

$$
a_{i j}= \begin{cases}1, & \text { if } p_{j} \text { covers } r_{i} \\ 0, & \text { otherwise }\end{cases}
$$

We want to solve the following constrained optimization problem (weighted set covering problem (WSC, in short)).

$$
\min _{x \in\{0,1\}^{n}} \sum_{j=1}^{n} x_{j} w_{j}, \quad \text { such that } \sum_{j=1}^{n} a_{i j} x_{j} \geq 1, \quad \text { for } i=1, \ldots, m .
$$


Table 1. Left: input covering matrix; position $(i, j)$ contains a 1 if protein $p_{j}$ occurs in the set of selected hits of read $r_{i}$, otherwise it contains a 0 . Right: proxygenes selected by the GWproxy are indicated with bigger fonts.
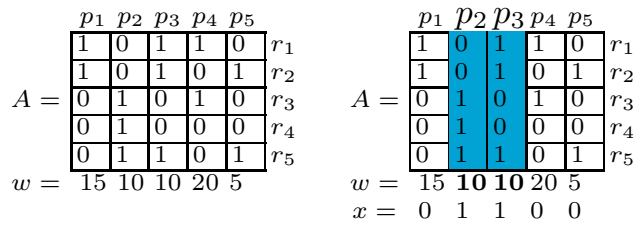

The variable $x_{j}$ indicates whether $p_{j}$ belongs to the solution $\left(x_{j}=1\right)$ or not $\left(x_{j}=0\right)$. The $m$ constraint inequalities are used to express the requirement that each read $r_{i}$ be covered by at least one protein. The weight $w_{j}$ specifies the cost of protein $p_{j}$.

Here a fast heuristic algorithm 2 for WSC 12 is applied to find a solution in an acceptable time. A solution corresponds to a subset of $P$ consisting of those proteins $p_{j}$ such that $x_{j}=1$. Each of the selected proteins is a proxygene. It represents the cluster consisting of those reads covered by that protein.

Example 2. We illustrate the application of GWproxy on the toy problem of Example 1. Let $w=\left(\begin{array}{lllll}15 & 10 & 10 & 20 & 5\end{array}\right)$ be the vector of protein weights. Then Table 1 (left part) shows the corresponding matrix $A \in\{0,1\}^{5 \times 5}$. Application of GWproxy outputs proteins $p_{2}, p_{3}$ (see Table 1 right part). The selected proteins correspond to the two clusters of reads $\left\{r_{3}, r_{4}, r_{5}\right\}$ and $\left\{r_{1}, r_{2}, r_{5}\right\}$, with $p_{2}$ and $p_{3}$ as associated proxygenes, respectively.

\subsection{EGWproxy}

The aim of the EGWproxy algorithm is to refine the clustering produced by GWproxy as follows. As we described before, each cluster that GWproxy outputs is represented by one protein. However, because of the short length of reads, and because in general the size of clusters is not very big (see analysis in [8]), it may well happen that more than one protein cover all the reads of a cluster.

Despite GWproxy selects only one of such proteins, among those having best score, each of the proteins covering a cluster can be considered as an equivalent representative of that cluster. Taking into account this fact, we can increase the robustness of GWproxy proxygenes choice method. For instance, for performing taxonomic analysis of cluster $C$, the following criterion can be used in order to decide which taxID to associate to $C$. The set $T$ of taxID's of the list of proteins that EGWproxy associates to $C$ is considered. Then the final taxID $t_{\text {fin }}$ of $C$ is computed as

$$
t_{\text {fin }}=\arg \min _{t \in T} \sum_{p \text { with taxID equal to } t} \frac{N_{p}}{w_{p}},
$$

${ }^{2}$ Publicly available at http://www.cs.ru.nl/ elenam 
where the operator arg min gives the element at which the objective function takes its minimum.

\subsection{Comparison of Algorithms}

GWproxy and LWproxy use different clustering heuristics: the first algorithm searches a clustering in a fixed searching space characterized by the sets of reads covered by each protein in $R$, while LWproxy constructs incrementally clusters of reads and of proteins. Furthermore, GWproxy scores proteins using bit and identities score, while LWproxy uses only bit score. Finally, GWproxy scores each protein globally, that is, using all the reads it covers, while LWproxy scores only the proteins of a cluster, where each protein is scored locally using the reads it covers that belong to that cluster.

Both EGWproxy and LWproxy associates to each cluster of reads one set of proteins. However, while LWproxy selects one protein as final representative of a cluster, EGWproxy employs an ensemble technique in order to exploit information of all the proteins of that set.

A drawback of LWproxy is that results may be affected by the choice of the read used in the first step of the algorithm, as illustrated by the following example.

Example 3. Consider the toy problem in Example 1. Suppose LWproxy selects $r_{5}$ as seed of the first cluster $C_{1}$. Then it adds all the other reads to $C_{1}$, since their best hit is in the list of hits of $r_{5} . P_{1}$ becomes equal to the entire set of proteins. Suppose for simplicity that all proteins have equal bit-score. Then LWproxy selects $p_{2}$ as proxygene, since it has highest cumulative bit-score. Hence, this clustering

While in the experiments here conducted this drawback does not seem to affect the results, it remains to be investigated whether this drawback does not affect results in general.

A drawback of GWproxy is that it outputs only one solution, while in general there may be more "optimal" clustering of reads. This is because the weighted set covering problem seeks one optimal solution, not the set of all optimal solutions. EGWproxy tries to overcome this drawback by using a post-processing step, followed by the application of an ensemble technique for merging multiple solutions. However, the post-processing step acts only on the set of proteins, while the clusters of reads remain those produced by GWproxy. It remains to be investigated whether application of ensemble techniques also at the level of clusters of reads can improve the performance of the method.

\section{Taxonomic Analysis of Metagenome Data}

We consider three complex metagenome datasets introduced in [5], called in the following M1, M2 and M3. These datasets were generated, respectively, from 9, 5 and 8 genome projects, sequenced at the Joint Genome Institute (JGI) using the 454 GS20 pyrosequencing platform that produces $\sim 100 \mathrm{bp}$ reads. From 
Table 2. Characteristics of the organisms used in the experiments: the identifier and name of the organism, the size of its genome and the total number of reads sampled (M1 dataset)

\begin{tabular}{|c|c|c|c|}
\hline Id. & Organism & genome size (bp) & reads sampled \\
\hline $\mathrm{a}$ & Clostridium phytofermentans ISDg & 4533512 & 4638 \\
\hline b & Prochlorococcus marinus NATL2A & 1842899 & 1866 \\
\hline c & Lactobacillus reuteri $100-23$ & 2174299 & 2371 \\
\hline d & Caldicellulosiruptor saccharolyticus DSM 8903 & 2970275 & 2950 \\
\hline e & Clostridium sp. OhILAs & 2997608 & 2934 \\
\hline $\mathrm{f}$ & Herpetosiphon aurantiacus ATCC 23779 & $6 \quad 605151$ & 6937 \\
\hline $\mathrm{g}$ & Bacillus weihenstephanensis KBAB4 & 5602503 & 4158 \\
\hline $\mathrm{h}$ & Halothermothrix orenii H 168 & 2578146 & 2698 \\
\hline $\mathrm{i}$ & Clostridium cellulolyticum $\mathrm{H} 10$ & 3958683 & 3978 \\
\hline
\end{tabular}

each genome project, reads were sampled randomly at coverage level $0.1 X$. The coverage is defined as the average number of times a nucleotide is sampled. This resulted in a total of 35230,28870 and 35861 reads, respectively.

Table 3 shows the names of the organisms and the number of reads generated for the M1 dataset. The reader is referred to 5. for a detailed description of all the datasets.

In our experiments we use the $\mathrm{NR}^{3}$ (non-redundant) protein sequence database as reference database for BLASTx. The parameters of the external software we used are set as follows. For BLASTx the default parameters were used. In all experiments we used Evalue cutoff $E=10^{-6}$. Moreover, WSCP was run with pre-processing $(-p)$, number of iterations equal to $1000(-x 1000)$, one tenth of the best actual cover used as starting partial solution $(-a 0.1)$, and 150 columns to be selected for building the initial partial cover at the first iteration $(-b 150)$. For lack of space, we refer to [12] for a detailed description of the WSCP program.

\subsection{Results}

We extract taxonomic information from each metagenome dataset as follows. For LWproxy and GWproxy each cluster of reads is represented by one protein. The taxID of such protein is used as taxonomic information of that cluster. For EGWproxy the list of proteins associated to each cluster is transformed into one taxID as described in Section 2.4.

In this way, the metagenomic data is transformed into a set of taxID's of proteins, one for each cluster of reads. Taxonomic information is then retrieved from the NCBI taxonomy (see http://www.ncbi.nlm.nih.gov/Taxonomy/). The NCBI Taxonomy database is a curated set of taxonomic classifications for all the organisms that are represented in GenBank. Each taxon in the database is associated with a numerical unique identifier called taxID. In the present analysis, the taxonomic information of these known proxygenes is used to determine the taxonomic content of the metagenomic data.

${ }^{3}$ Publicly available at ftp://ftp.ncbi.nlm.nih.gov/blast/db 
We visualize the resulting taxonomic information in two ways.

- Graph representation of taxonomic distribution of reads, as done e.g. in [14. Here analysis at the taxonomic level of phylum and class is performed, where resulting taxa containing less than 100 reads are discarded.

- Histogram of phylogenetic identities, as done e.g. in [13]. Shown are the percentages of the total of identifiable hits assigned to the phylogenetic groups obtained by means of the taxID of the proxygenes. Here analysis at the class taxonomic level is performed.

We apply the above techniques to the proxygenes and taxid obtained from the considered algorithms, as well as to the known taxid's of the original metagenome data sets, provided by the producers of the benchmark data [5]. We use these latter results as "golden truth" (GT in short) to evaluate the methods.

For lack of space, we show graphs of the taxonomic distribution at phylum and class level only for M1 in Figure 1. Results indicate satisfactory consensus

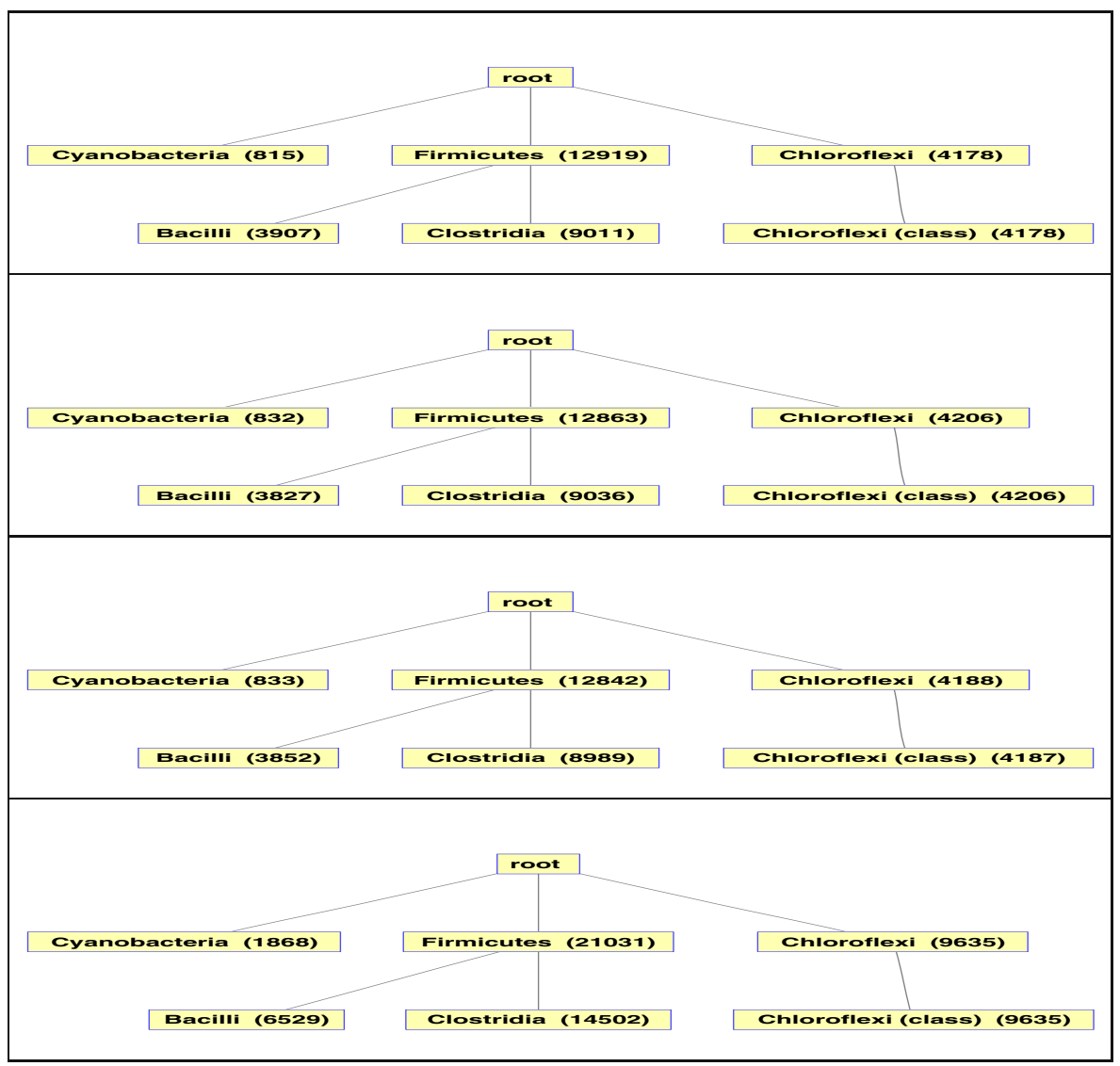

Fig. 1. Phylogenetic graph for M1. From top to bottom: LWproxy, GWproxy, EGWproxy and "Golden Truth". 

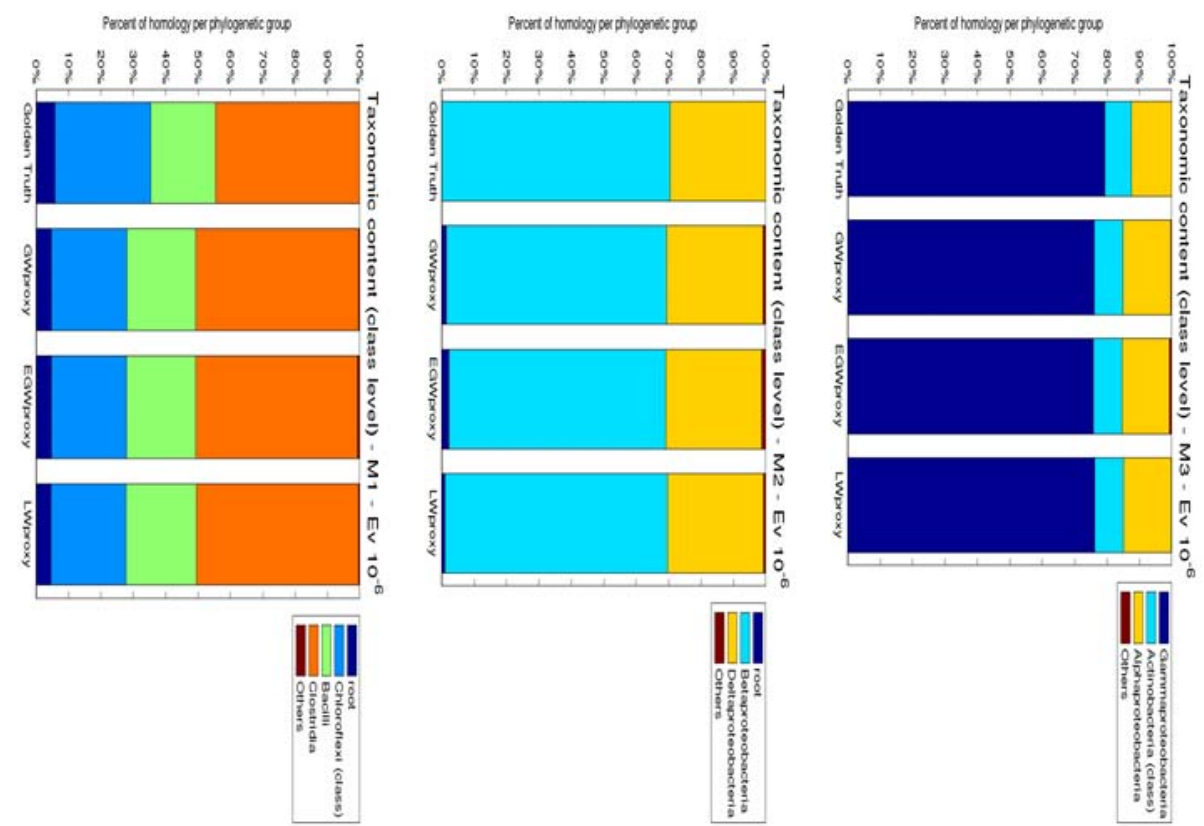

Fig. 2. Taxonomic distribution at taxonomic class level of the three datasets. From left to right: M1, M2 and M3. From top to bottom: "golden truth", GWproxy, EGWproxy and LWproxy.

among the three methods, yielding similar type of graphs. The methods give graphs with the same structure of the "golden truth" for M1 and M3 datasets. For M2, instead, GWproxy and EGWproxy give graphs with a phylum subtree (Streptophyta) that does not appear in the "golden truth"; anyway they are made by just $1 \%$ and $2 \%$ of the covered reads, respectively.

Histograms of phylogenetic identities at the class level for the three data sets are shown in Figure2 The results achieved by the three methods are almost identical.

\section{Conclusion and Future Work}

In this paper we compared three methods for clustering reads and their application to taxonomic analysis of metagenome data. We discuss advantages and drawbacks of the methods and applied them to perform taxonomic analysis of three real-life metagenome datasets with known taxonomic content.

Results of such analysis indicate satisfactory consensus of all the three methods, and very good performance with respect to taxonomic distribution and phylogenetic content. Unfortunately, a drawback of LWproxy is that results can be affected by the choice of the read used in the first step of the algorithm, as shown in Example 3. While in the experiments here conducted this drawback 
does not seem to affect the results, it remains to be investigated whether this drawback does not affect results in general. The algorithms EGWproxy can potentially refine the proxygenes selection made by GWproxy despite we found no evidence of this fact in our analysis yet.

In future we intend to introduce a statistical test for measuring significance of taxonomic assignment, in order to discard assignments possibly due to the composition of the reference proteome database used when applying BLASTx. Such a test will consider not only the number of reads assigned to a taxa, but also the divergence of their proxygenes as well as the nucleotide composition of the reads.

Acknowledgements. We would like to thank Mavrommatis Konstantinos for providing the datasets in [5] as well as useful information about such data.

\section{References}

1. Yooseph, S., et al.: The Sorcerer II global ocean sampling expedition: Expanding the universe of protein families. PLoS Biol. 5(3), e16 (2007)

2. McHardy, A., Rigoutsos, I.: What's in the mix: phylogenetic classification of metagenome sequence samples. Current Opinion in Microbiology 10, 499-503 (2007)

3. Raes, J., Foerstner, K., Bork, P.: Get the most out of your metagenome: computational analysis of environmental sequence data. Current Opinion in Microbiology 10, 490-498 (2007)

4. Li, W., Wooley, J., Godzik, A.: Probing metagenomics by rapid cluster analysis of very large datasets. PLoS One 3(10) (2008)

5. Dalevi, D., Ivanova, N., Mavromatis, K., Hooper, S., Szeto, E., Hugenholtz, P., Kyrpides, N., Markowitz, V.: Annotation of metagenome short reads using proxygenes. Bioinformatics 24(16) (2008)

6. Pop, M., Phillippy, A., Delcher, A., Salzberg, S.: Comparative genome assembly. Briefings in Bioinformatics 5(3), 237-248 (2004)

7. Chan, C., Hsu, A., Tang, S., Halgamuge, S.: Using growing self-organising maps to improve the binning process in environmental whole-genome shotgun sequencing. Journal of Biomedicine and Biotechnology (2008)

8. Folino, G., Gori, F., Jetten, M.S.M., Marchiori, E.: Clustering metagenome short reads using weighted proteins. In: EvoBIO 2009. LNCS, vol. 5483, pp. 152-163. Springer, Heidelberg (2009)

9. Korf, I., Yandell, M., Bedell, J.: BLAST. O'Reilly \& Associates, Inc., Sebastopol (2003)

10. Madden, T.: 16. In: The BLAST Sequence Analysis Tool, Bethesda, MD (2002)

11. Altschul, S., Gish, W., Miller, W., Myers, E., Lipman, D.: Basic local alignment search tool. J. Molecular Biology 215(3), 403-410 (1990)

12. Marchiori, E., Steenbeek, A.: An evolutionary algorithm for large scale set covering problems with application to airline crew scheduling. In: Oates, M.J., et al. (eds.) EvoIASP 2000, EvoWorkshops 2000, EvoFlight 2000, EvoSCONDI 2000, EvoSTIM 2000, EvoTEL 2000, and EvoROB/EvoRobot 2000. LNCS, vol. 1803, pp. 367-381. Springer, Heidelberg (2000)

13. Biddle, J.F., et al.: Metagenomic signatures of the Peru margin subseafloor biosphere show a genetically distinct environment. PNAS (105), 10583-10588 (2008)

14. Venter, J., et al.: Environmental genome shotgun sequencing of the sargasso sea. Science (304), 66-74 (2004) 